\title{
Anomalous dilepton production as precursory phenomena of color superconductivity
}

\author{
Toru Nishimura, ${ }^{a, *}$ Masakiyo Kitazawa ${ }^{a, b}$ and Teiji Kunihiro ${ }^{c}$ \\ ${ }^{a}$ Department of Physics, Osaka University, \\ 560-0043, Toyonaka, Osaka, Japan \\ ${ }^{b}$ J-PARC Branch, KEK Theory Center, Institute of Particle and Nuclear Studies, KEK, 203-1, \\ 319-1106, Shirakata, Tokai, Ibaraki, Japan \\ ${ }^{c}$ Yukawa Institute for Theoretical Physics, Kyoto University, \\ 606-8502, Kyoto, Japan \\ E-mail: nishimura@kern.phys.sci.osaka-u.ac.jp, \\ kitazawa@phys.sci.osaka-u.ac.jp, kunihiro@yukawa.kyoto-u.ac.jp
}

We explore how the dilepton production rate is modified by the diquark fluctuations that form welldeveloped collective modes near but above the critical temperature of color superconductivity. Through the analysis of the photon self-energy called the Aslamasov-Larkin, Maki-Thompson and density of states terms which are known in the metalic superconductivity, it is shown that the diquark fluctuation mode affects the photon self-energy significantly and thereby cause to anomalously enhance the dilepton production rate in the low invariant-mass region.

\footnotetext{
*** Particles and Nuclei International Conference - PANIC2021 ***

*** 5 - 10 September, 2021 ***

*** Online ***
}

${ }^{*}$ Speaker 


\section{Introduction}

It is expected that rich physics in high baryon-density matter at finite temperature will be revealed by experimental programs in relativistic heavy-ion collisions (HIC) such as the beamenergy scan program at RHIC, HADES and NA61/SHINE, as well as the future plans at FAIR, NICA and J-PARC-HI. In these proceedings, we investigate the observability of the color superconductivity (CSC) [1] in these experiments through their precursory phenomena that are developed near but above the critical temperature $T_{c}$ of the CSC $[2,3]$. For this purpose, we investigate the effect of the diquark fluctuations on the dilepton production rate by extending the theory of the paraconductivity in metals [3]. We show that the diquark fluctuations lead to anomalous enhancement of the dilepton production rate at low invariant-mass region near $T_{c}$.

\section{Diquark fluctuations}

The 2-flavor color-superconductivity (2SC) is expected to be realized at relatively low densities, which may be achieved by HIC experiments. Thus, we employ the massless 2-flavor NJL model

$$
\mathcal{L}=\bar{\psi} i \gamma_{\mu} \partial^{\mu} \psi+G_{S}\left[(\bar{\psi} \psi)^{2}+\left(\bar{\psi} i \gamma_{5} \vec{\tau} \psi\right)^{2}\right]+G_{C}\left(\bar{\psi} i \gamma_{5} \tau_{2} \lambda_{A} \psi^{C}\right)\left(\bar{\psi}^{C} i \gamma_{5} \tau_{2} \lambda_{A} \psi\right),
$$

where $\tau_{2}\left(\gamma_{A=2,5,7}\right)$ is the antisymmetric component of the Pauli (Gell-Mann) matrices for the flavor $S U(2)_{f}$ (color $\left.S U(3)_{c}\right)$, and $\psi^{C}(x) \equiv C \bar{\psi}^{T}(x)$ with $C=i \gamma_{2} \gamma_{0}$. The scalar coupling constant $G_{S}=5.01 \mathrm{MeV}^{-2}$ and the three-momentum cutoff $\Lambda=650 \mathrm{MeV}$ are determined so as to reproduce the pion decay constant $f_{\pi}=93 \mathrm{MeV}$ and the chiral condensate $\langle\bar{\psi} \psi\rangle=(-250 \mathrm{MeV})^{3}$ in the chiral limit [2]. The diquark coupling constant is set to $G_{C}=0.6 G_{S}$.

In Ref. [2], it has been pointed out that the diquark fluctuations form the collectivity with a significant strength above but near the critical temperature $T_{c}$ of the 2SC. The propagator $\Xi(q)$ of the diquark fluctuations in the random-phase approximation (RPA) is in the imaginary-time formalism given by

$$
\Xi(q)=\frac{G_{C}}{1+G_{C} Q(q)}, \quad Q(q)=-2 N_{f}\left(N_{c}-1\right) \int \frac{d^{4} p}{(2 \pi)^{4}} \operatorname{Tr}\left[\mathcal{G}_{0}(q-p) \mathcal{G}_{0}(p)\right],
$$

where $Q(q)=\boldsymbol{Q}\left(\boldsymbol{q}, i v_{n}\right)$ is the one-loop $q q$ correlation function; $\mathcal{G}_{0}(p)=\mathcal{G}_{0}\left(\boldsymbol{p}, i \omega_{m}\right)=1 /\left[\left(i \omega_{m}+\right.\right.$ r) $\left.\gamma_{0}-\boldsymbol{q} \cdot \gamma\right]$ is the free quark propagator, $\omega_{m}\left(v_{n}\right)$ the Matsubara frequency for fermions (bosons), and $\operatorname{Tr}$ the trace over the Dirac indices. The retarded propagator is obtained by taking the analytic continuation $\Xi^{R}(\boldsymbol{q}, \omega)=\Xi\left(\boldsymbol{q}, i v_{n} \rightarrow \omega+i \eta\right)$.

We remark that the equality $\left[\Xi^{R}(\mathbf{0}, 0)\right]^{-1}=0$ is satisfied at $T=T_{c}$ determined by the MFA, which is the Thouless criterion for determining the critical temperature of the second-order phase transition. The criterion shows that $\Xi^{R}(\boldsymbol{q}, \omega)$ has a pole at the origin at $T=T_{c}$, and hence the diquark fluctuations have the properties of the soft mode [2]. This fact also allows us to approximate the propagator of the fluctuations in the low energy-momentum near but above $T_{c}$ as

$$
\Xi^{R}(\boldsymbol{q}, \omega)=1 /\left[c_{0} \omega+c_{1} \boldsymbol{q}^{2}+a\right],
$$

where $a=G_{C}\left[\Xi^{R}(\mathbf{0}, 0)\right]^{-1}, c_{0}=G_{C}(\partial / \partial \omega)\left[\Xi^{R}(\mathbf{0}, 0)\right]^{-1}$ and $c_{1}=G_{C}\left(\partial / \partial \boldsymbol{q}^{2}\right)\left[\Xi^{R}(\mathbf{0}, 0)\right]^{-1}$ from $\Xi^{R}(\boldsymbol{q}, \omega)$ obtained in the NJL model. This approximation is called the time-dependent Ginzburg-Landau (TDGL) approximation in the literature. In Ref. [2], it has been shown that the behavior of $\Xi^{R}(\boldsymbol{q}, \omega)$ is reproduced over wide ranges of $\omega, \boldsymbol{q}^{2}$ and $T\left(>T_{c}\right)$. 


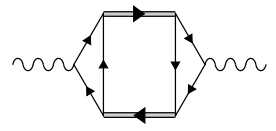

(a)

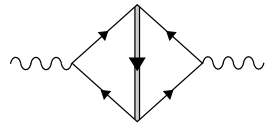

(b)

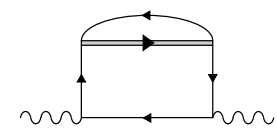

(c)

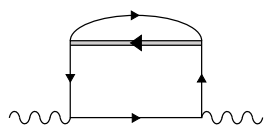

(d)

Figure 1: Diagrammatic representations of the Aslamasov-Larkin (a), Maki-Thompson (b) and density of states $(\mathrm{c}, \mathrm{d})$ terms. The wavy and double lines are photons and diquark fluctuations, respectively.

\section{Modification of photon self-energy by diquark fluctuations}

The dilepton production rate is given in terms of the retarded photon self-energy $\Pi^{R \mu \nu}(k)$ as

$$
\frac{d^{4} \Gamma}{d^{4} k}=\frac{\alpha}{12 \pi^{4}} \frac{1}{k^{2}} \frac{1}{e^{\beta \omega}-1} g_{\mu \nu} \operatorname{Im} \Pi^{R \mu \nu}(k),
$$

where $k=(\boldsymbol{k}, \omega)$ is the four momentum of the photon and $\alpha$ is the fine structure constant. To evaluate the effects of the diquark fluctuations on the dilepton production rate, the modification of photon self-energy due to $\Xi(\boldsymbol{q}, \omega)$ needs to be investigated.

We start from the one-loop diagram of $\Xi\left(\boldsymbol{q}, i v_{n}\right)$, which is the lowest contribution of diquark fluctuations to the thermodynamic potential. The photon self-energy is then constructed by attaching electromagnetic vertices at two points of quark lines in the thermodynamic potential. One then obtains four types of diagrams shown in Fig. 1. These diagrams are called the Aslamasov-Larkin (AL) (Fig. 1(a)), Maki-Thompson (MT) (Fig. 1(b)) and density of states (DOS) (Fig. 1(c, d)) [4] terms, respectively, in the theory of metallic superconductivity. Each contribution to the photon self-energy, $\Pi_{\mathrm{AL}}^{\mu v}(k), \Pi_{\mathrm{MT}}^{\mu v}(k)$ and $\Pi_{\mathrm{DOS}}^{\mu v}(k)$, respectively, is given by

$$
\begin{aligned}
& \Pi_{\mathrm{AL}}^{\mu v}(k)=4 N_{c} T \sum_{n} \int \frac{d^{3} q}{(2 \pi)^{3}} \Gamma^{\mu}(q, q+k) \Xi(q+k) \Gamma^{v}(q+k, q) \Xi(q), \\
& \Pi_{\mathrm{MT}(\mathrm{DOS})}^{\mu v}(k)=2 N_{c} T \sum_{n} \int \frac{d^{3} q}{(2 \pi)^{3}} \Xi(q) R_{\mathrm{MT}(\mathrm{DOS})}^{\mu \nu}(q, k),
\end{aligned}
$$

where $\Gamma^{\mu}(q, q+k)$ and $R^{\mu v}(q, k)=R_{\mathrm{MT}}^{\mu v}(q, k)+R_{\mathrm{DOS}}^{\mu v}(q, k)$ satisfies the WT identity as follows,

$$
k_{\mu} \Gamma^{\mu}(q, q+k)=\Xi^{-1}(q+k)-\Xi^{-1}(q), \quad k_{\mu} R^{\mu \nu}(q, k)=2\left[\Gamma^{\nu}(q-k, q)-\Gamma^{\nu}(q, q+k)\right] .
$$

The total photon self-energy is then given by $\Pi^{\mu \nu}(k)=\Pi_{\text {free }}^{\mu v}(k)+\Pi_{\mathrm{AL}}^{\mu \nu}(k)+\Pi_{\mathrm{MT}}^{\mu v}(k)+\Pi_{\mathrm{DOS}}^{\mu v}(k)$. One can explicitly check that this photon self-energy satisfies the WT identity using Eq. (7).

To calculate the diagrams in Fig. 1, we approximate $\Gamma^{\mu}(q, q+k)$ and $R^{\mu \nu}(q, k)$ similarly to Eq. (3). To obtain the dilepton production rate Eq. (4), the calculation of the spatial components of $\Pi^{R \mu \nu}(k)$ is sufficient since $\Pi^{00}(k)$ is expressed in terms of the longitudinal part as $\Pi^{R 00}(k)=$ $\boldsymbol{k}^{2} \Pi^{R 11}(k) / k_{0}^{2}$ with $k=\left(k_{0},|\boldsymbol{k}|, 0,0\right)$ from the WT identity. Substituting Eq. (3) into Eq. (7) and comparing the lowest order terms of $\boldsymbol{k}$, we obtain the spacial components of vertices as follows,

$$
\Gamma^{i}(q, q+k)=c_{1}(2 q+k)^{i}, \quad R^{i j}(q, k)=-4 c_{1} k^{i} k^{j} / \boldsymbol{k}^{2} .
$$

One finds that the imaginary part of $\Pi_{\mathrm{MT}}^{R i j}(q)+\Pi_{\mathrm{DOS}}^{R i j}(q)$ calculated with Eqs. (3) and (8) vanishes. Therefore, the MT and DOS terms do not contribute to the dilepton production rate. This result is in accordance with the case of the metallic superconductivity [4]. 

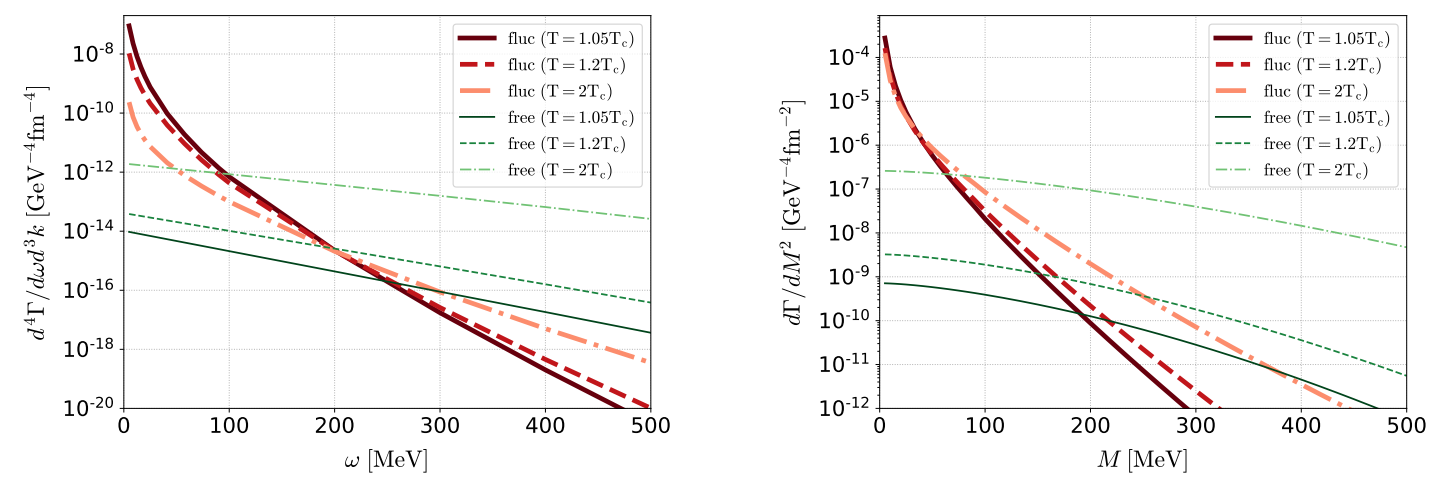

Figure 2: Dilepton production rates at $\mu=350 \mathrm{MeV}$ per unit energy $\omega$ at $\boldsymbol{k}=0$ (left) and per invariant mass $M$ (right). In each panel, the thick (red) lines are the contribution of diquark fluctuations $\Pi_{\text {fluc }}^{\mu \nu}(k)$, and the thin (green) lines are the results for the free quark gas. The solid, dashed and dash-dotted lines are the results for $T=1.05 T_{c}, 1.2 T_{c}$ and $2 T_{c}$.

\section{Numerical results and summary}

Figure 2 represents the dilepton production rate at the quark chemical potential $\mu=350 \mathrm{MeV}$ for three temperatures above $T_{c}$. The left panel is the production rate per unit energy and momentum $d^{4} \Gamma / d^{4} k$ at $\boldsymbol{k}=0$. The thick lines are the results due to diquark fluctuations, while the thin lines are the ones of the free quark gas. One sees that the production rate from diquark fluctuations is anomalously enhanced in the relatively small $\omega$ region in comparison with the free quark gas for $T \lesssim 2 T_{c}$, and this enhancement becomes more pronounced as $T$ approaches $T_{c}$. This result is expected through the soft property of the diquark fluctuations. The right panel is the invariant-mass $(M)$ spectrum $d \Gamma / d M^{2}$, which is more useful in comparison to experiments. One finds that the rate is increasing at small $M$, whereas the $T$ dependence of the increase becomes milder than $d^{4} \Gamma / d^{4} k$.

In this report, we studied how diquark fluctuations affects the dilepton production near but above $T_{c}$ of the $2 \mathrm{SC}$. The modification of the photon self-energy due to diquark fluctuations are investigated through the analysis of the Aslamasov-Larkin, Maki-Thompson and density of states terms with the TDGL approximation for the diquark propagator and vertices. The enhancement of the dilepton production rate up to $T \simeq 2 T_{c}$ would allow us to detect this signal in the future HIC experiments even when the CSC itself cannot be observable in these experiments.

\section{References}

[1] M. G. Alford, A. Schmitt, K. Rajagopal and T. Schäfer, Rev. Mod. Phys. 80 (2008) 1455.

[2] M. Kitazawa, T. Koide, T. Kunihiro and Y. Nemoto, Phys. Rev. D 65 (2002) 091504; ibid., Phys. Rev. D 70 (2004) 056003; ibid., Prog. Theor. Phys. 114 (2005) 117.

[3] T. Kunihiro, M. Kitazawa and Y. Nemoto, PoS CPOD07 (2007) 041.

[4] A. I. Larkin and A. A. Varlamov, Springer Berlin Heidelberg (2008). 